\title{
CHANGING BACTERIAL ORGANISMS AND ANTIMICROBIAL RESISTANCE PATTERN AMONG HOSPITALIZED COMMUNITY ACQUIRED PNEUMONIA PATIENTS IN A TERTIARY CARE CENTER
}

Raveendra Kodur Ramamurthy. Ravi Ningappa, Jayachandra

1. Associate Professor. Department of Medicine, Bangalore Medical College \& Research Institute, Bangalore,
2. Assistant Professor. Department of Radiology, Bangalore Medical College \& Research Institute, Bangalore,
3. Assistant Professor. Department of Medicine, Bangalore Medical College \& Research Institute, Bangalore,

\section{CORRESPONDING AUTHOR:}

Dr. Raveendra Kodur Ramamurthy,

Associate Professor of Medicine, K. R. Road,

Chamarajpet, New Tharagupet, Bangalore,

Karnataka - 560002

E-mail: dr.krravendra@gmail.com

ABSTRACT: BACKGROUND: Most of the times a Community Acquired Pneumonia (CAP) patient is being treated with empirical antibiotics by best guess method by the clinician.MDR strains are being reported from c/s reports and Gram negative rods are fast increasing both in the etiology and mortality of CAP patients.

\section{AIM OF STUDY:}

1. To find out the profile of different bacteria causing CAP at a tertiary hospital.

2. To know the treatment outcome \& drug resistance pattern among culture positive CAP patients.

MATERIALS \& METHODS: 450 CAP patients admitted \& treated between Jan. to Dec.2012 at Victoria Hospital, Bangalore were included in this hospitalized cross sectional study. All relevant investigations including sputum c/s were done.

RESULTS: Positive culture reports were obtained in 165(36.33\%) out of 450 patients Following are the isolates-Strept.pnemoniae $32.7 \%$, Klebsiella 18.2\%, Staph.aureas 10.9\%, Psedomonas 10.9\%, E.coli 5.4\%, Acinetobacter 7.3\%, H.influenza 5.4\%, Mixed growth 10\%. 76\% of patients recovered from CAP, $6 \%$ went against medical advice, $6.6 \%$ showed unresolved pneumonia \& deaths in $5.33 \%$ of patients. High degree of resistance was noted to many antibiotics including to latest cephalosporins.

CONCLUSION: This study highlights the importance of culture tests and selection of proper antibiotics and to avoid misuse \& abuse of higher antibiotics to prevent the emergence of MDR strains. An antibiotic policy at every level may help.

KEYWORDS: Community acquired pneumonia (CAP), Pneumonia, Multidrug resistance (MDR).

INTRODUCTION: Community acquired pneumonia (CAP) is a pneumonia that has been acquired in a community in a patient who has not been hospitalized within 14 days prior to onset of symptoms ${ }^{1}$. Pneumonia carries a significant mortality and morbidity all over the world despite the availability of better and more potent antibiotics and improved supportive care. Pneumonia is often misdiagnosed and commonly treated inadequately or over treated with 
misuse of potent antibiotics. Ideally treatment for CAP should be based on culture and sensitivity report of the organism identified by culture or serological methods. However in a majority of patients especially in our country, treatment is empirical and based on surveillance data or 'best guess' method. So, antibiotics are being prescribed after considering the epidemiological data, background history of patients and clinical presentation.

Though there is decreased mortality with implementation of guidelines ${ }^{2}$, widespread antibiotic misuse and abuse have been reported at all levels from patients to pharmacists to consultants. There is rapid shift in the bacteriological profile causing CAP with Streptococcus pneumonia as commonest ${ }^{3}$ to gram negative rods $^{3,4}$.

Multidrug resistance (like Beta lactams, macrolides, doxycycline, fluoroquinolones and to latest cephalosporin) is an emerging problem and complicates the management of CAP1-5. Hence identifying changing bacterial flora and drug sensitivity play an important role in the management of CAP.

MATERIALS AND METHODS: The study was designed as a hospital based cross sectional study conducted at Victoria hospital, a tertiary care center at Central Bangalore attached to BMCRI from January to December 2012, for a period of one year. A total of 478 patients diagnosed based on clinical presentation, radiological findings and bacteriological profile, were treated as inpatients under the department of medicine. Due to the non-availability of vital statistics (24) and pulmonary Tuberculosis (sputum positive- 4 patients), 28 patients were excluded and only 450 were included in the study. All the patients after detailed history and clinical examination underwent routine blood investigations including LFT, RFT, Chest X ray, Ultrasound abdomen, sputum Gram staining, AFB and Culture Sensitivity, HIV testing and pulse oximetry.

While anaerobic sputum culture, fungal stains were not done, blood culture sensitivity, CT chest were performed only in some limited non responding patients. All the patients were treated empirically with broad spectrum antibiotics (changed after getting the culture sensitivity report). Comorbid conditions of the patients were taken into consideration while managing CAP. Most of the patients were discharged from the hospital after completion of the antibiotic course and/ or resolution of symptoms. Unresolved CAP patients continued for investigations and management at the hospital.

OBSERVATIONS AND RESULTS: A total of 450 patients were included in the study, out of which $267(59.33 \%)$ were males and $183(40.66 \%)$ were females, with a male: female ratio $\sim 3: 2$. Majority of the patients 296 belonged to the age group 40-70years (65.77\%).Fever 414(92\%), cough 432(96\%), breathlessness 387(86\%) were the most common symptoms. Though patients were seen around the year, majority (54.66\%) was seen from July to October. $378(84 \%)$ patients had symptoms of less than one week. Important comorbid conditions were smoking 46\%(males), alcohol abuse 28\%(males), diabetes 16.6\%, COPD 8.4\%(males), Hypertension $12 \%$, anemia $26 \%, 4.44 \%$ (20 patients) had HIV positive status, 2.66\%(12 patients) had HBsAg positive status.

On examination, $86 \%$ had fever, $82 \%$ had tachypnea, $72 \%$ had tachycardia and rales were seen in $84 \%$ patients. On chest X ray, $42 \%$ had right lower lobe, $21 \%$ had left lower lobe, $12 \%$ had right upper lobe, $5 \%$ had left upper lobe, $2 \%$ had right middle lobe and $18 \%$ had multilobar lesions. Patients were classified using PORT scoring guidelines to know the mortality 5 as a predictor rule and $56 \%$ fell under Category IV, $34 \%$ in category III and $18 \%$ in category V. 
$117(26 \%)$ CAP patients were already exposed to at least one antibiotic before admission, with $36(8 \%)$ patients having completed at least one full course of antibiotics. Positive cultures were obtained in $165(36.3 \%)$ patients, out of which Streptococcus pneumonia in 54(32.7\%),Staphylococciin18 (10.9\%), Klebsiella 30(18.2\%), Pseudomonas18 (10.9\%), E.coli 9(5.4\%), Acinetobacter 12(7.3\%), H Influenza 9(5.4\%). All the patients were started on antibiotics empirically mainly ceftriaxone $(78 \%)$, cefuroxime $(2 \%)$, Ceftriaxone+sulbactam (2\%), Amoxcyllin+clavulinicacid (2\%), Ciprofloxacin+gentamicin (4\%), Penicillin+gentamicin (2\%), others $(10 \%)$.

$36(21.8 \%)$ culture positive CAP patients required a change in antibiotics after culture sensitivity report. Of the culture negative patients (285), 40(14.04\%) required a change in antibiotic at the end of fifth day and some patients were treated with multiple antibiotics. Metronidazole was given to all suspected anaerobic infections.

To sum up216(48\%) patients recovered in a week's time and were discharged, $126(28 \%)$ patients recovered in less than two weeks' time,27(6\%) patients stayed for more than two weeks. 16 patients(3.55\%) required ICU support, 11(2.44\%) went against medical advice and 30 (6.66\%) showed unresolved pneumonia with deaths occurring in 24 patients( $5.33 \%)$.

There were 24 deaths noted in the study and the causes of death were septicemia and multi organ dysfunction in 10(41.66\%), acute renal failure in 4(16.66\%), ARDS in 4(16.66\%), PCP Pneumonia in 4(16.66\%) and DKA in 2(8.33\%) patients.

Resistance to streptococcus pneumonia was seen in up to $49 \%$ for amox-clav, $70 \%$ for azithromycin, 55\% for ciprofloxacin, 79\% for doxycycline, and 59\% for ceftriaxone. Resistance to Klebsiella species was found to be $59 \%$ for amox-clav, $82 \%$ for gentamycin, $54 \%$ for amikacin, $82 \%$ for ciprofloxacin, and $70 \%$ for ceftriaxone. Resistance to pseudomonas was observed in $79 \%$ for ciprofloxacin, $52 \%$ for amikacin, $72 \%$ for ceftriaxone, $50 \%$ for netilmycin, $40 \%$ for pipercillin and $29 \%$ for meropenam.

DISCUSSION: The management of CAP is fast changing and challenging in the presence of multidrug resistance of the organism, changing bacterial flora, and empirical treatment is started before asking for sputum culture sensitivity testing. In only $50 \%$ cases sputum culture yielded organisms with Streptococcus pneumonia as the commonest one ${ }^{5,6}$. The ATS statement describes the possibility of atypical pathogens (C.pneumoniae, M.pneumonia and L.pneumophila) either infecting or co-infecting with CAP and therefore recommends therapy to account for this possibility5. If patients with CAP require admission to the ICU one must consider S.pneumoniae, Legionella and enteric gram negative bacteria as organism responsible for the infection 3, 6, 7. Pseudomonas aeruginosa is considered in some severe CAP patients requiring ICU support and it requires a different treatment regimen ${ }^{3}$. Of the 450 patients of CAP included in the study, majority were males(59.33\%), and majority belonged to the age group 40-70 years(65.77\%). 84\% had acute symptoms and great majority had all three classical symptoms of fever, cough and breathlessness. MajorComorbid conditions noted were smoking (46\% of males), alcohol intake (28\% of males), diabetes (16.6\%), COPD (18.4\% of males), Hypertension (12\%), anemia (26\%). As many as $6.6 \%$ patients satisfied the criteria for unresolved pneumonia and $90 \%$ of them had at least one comorbid condition. Jay .S reported that the common conditions associated with delayed resolution are advanced age, COPD and alcoholism $^{8}$. Roson.B. et al have analyzed the independent factors associated with early treatment failure, were old age, multilobar pneumonia, PORT score more than 90, Legionella 
pneumonia, Gram negative pneumonia and discordant antimicrobial therapy9. In our study Gram staining was done in only 39\% patients and culture was positive in $36.3 \%$ patients. Poor culture positive rates are partly due to early exposure to antibiotics (up to 25\% patients had exposure to at least one antibiotic prior to admission), and probably due to the poor sputum specimen $(16 \%$ were poor specimen as reported by the microbiologist; later sputum samples were sent only in non-responding cases. Regional variations in the etiological agents of CAP are noted in India. Streptococcus pneumonia is the commonest organism in Shimla ${ }^{10}$ and Delhi ${ }^{11}$, Pseudomonas aeruginosa in blood culture positive CAP patients in Ludhiana and S. aureus followed by E.coli and Klebsiella in a study at Sher I Kashmir Institute of Medical Sciences ${ }^{12}$. In our study still Streptococcus pneumonia is the commonest organism in culture positive CAP but in(approx. 38\%) of cases Gram negative organisms were isolated and quite a few of them had developed complications requiring ICU support, and were associated with multidrug resistance and early deaths. Though ATS does not recommend routine sputum culture with Gram stain because in up to $40-50 \%$ cases a pathogen could not be identified ${ }^{13}$.

In the present study $48 \%$ patients responded to first antibiotic mainly ceftriaxone and antibiotics were changed in $20 \%$ of culture positive patients (to appropriate antibiotics) and $16 \%$ of culture negative patients (due to worsening of infection).

$21 \%$ of S.pneumoniae, $44.6 \%$ Klebsiella, $61.2 \%$ Pseudomonas, $58 \%$ S.aureus, $72.2 \%$ of E.coliwas resistant to more than three antibiotics.

$60 \%$ of the unresolved pneumonia patients, $40 \%$ patients with late resolution and $22 \%$ of patients who died had history of frequent usage of antibiotics (used more than 4 different antibiotics in the last 6 months). Gram negative bacilli were isolated in majority of the unresolved pneumonia (60\%) and in patients who died due to CAP (58.33\%).

The treatment of culture negative CAP patients were demanding and challenging as they had prolonged stay (16vs 11), more ICU support (9vs7), require a change in antibiotics (40vs36), unresolved pneumonia (19vs10), deaths (16vs8) when compared to culture positive cases.

The most practiced guidelines for the treatment of CAP include ATS ${ }^{13}$ and BTS ${ }^{4}$ and from Indian perspective are from PGIMER 14, Chandigarh. Hence it is better to adhere to some guidelines than best guess methods to prescribe antibiotics. Early administration of proper antibiotics with confirmed diagnosis of $\mathrm{CAP}^{15}$ is seen to decrease the mortality. In our study a lot of time was wasted by the patient to reach the hospital $(90 \%$ took more than 5 days of symptoms and $26 \%$ had exposed to some antibiotic and then after empirical treatment up to $36 \%$ patients needed a change in the antibiotics either after a $\mathrm{C} / \mathrm{S}$ report or after a clinical failure state). Hence much delay occurred for the initiation of proper antibiotics in the treatment of CAP.

CONCLUSION: CAP is a febrile infection, is fast changing in its presentation, risk factors/ comorbid conditions, bacterial flora and treatment outcome to the routine antibiotic usage. In the days of drug resistance (MDR varieties) and changing organisms, pathogen directed therapy is the order of the day by identifying the organism by reliable microbiological methods. Hence routine Gram staining and culture tests will help to minimize antibiotic misuse, to decrease hospital stay, to decrease the economic burden and to decrease mortality. All unresolved pneumonias should be thoroughly investigated for a specific cause and later for proper treatment. It is high time to have a policy in the antibiotic usage at different levels- hospital, district, state, and country-to prevent the emergence of MDR strains. Research in the antibiotic 
field for new antibiotics is as important as to preserve the existing antibiotics. This study highlights the importance of proper antibiotic for proper organism identified for the management of CAP.

\section{REFERENCES:}

1. Bartlett J, Dowell S, Mandell L, et al. practice guidelines for the management of community acquired pneumonia in adults. Clin Infect Dis 2000; 31:347-382.

2. Dean NC, Silver MP, Bateman KA, James B, Hadlock CJ, Hale D. Decreased mortality after implementation of a treatment guideline for community acquired pneumonia. Am J Med 2001; 110:451-7.

3. Lionel A. Mandell, Richard G. Wunderink, Antonio Anzueto, Cynthia G. Whitney et al. Infectious Diseases Society of America/ American Thoracic Society Consensus Guidelines on the Management of CAP in adults: ID 2007:44(Suppl 2)

4. Wei Shen Lim, Simon Baudouin, Robert George et al. The British Thoracic Society Guidelines for the management of CAP in adults Update 2009. Thorax VOL 64 Supplement III.

5. Fine MJ, Auble TE, Yealy DM, Hanusa BH, Weissfeld LA, singer DE et al. A prediction rule to identify low risk patients with CAP. N Engl J Med 1997;336:243-250.

6. M. Woodhead, F. Blasi, S.Ewig, G.Huchon, M.Ieven,A. Ortqvist et al. guidelines for the management of adult lower respiratory tract infections: EurRespir J 2005;26:1138-1180.

7. Mandell LA, Bartlett JG, Dowell SF, FILE TM Jr, Musher DM, Whitney C; Infectious Diseases Society of America. Update of Practice Guidelines for the practice of CAP in immunocompetent adults. Clin Infect Dis 2003;37:1405-1433.

8. Jay SJ, Johnson WG Jr, Pierce AK. The radiographic resolution of Streptococcus pneumonia. N Engl J Med 1975;293:798-801.

9. RosonB,Carratala J, Sabe NF, Tubau F, Manresa F, Gudiol F. causes and factors associated with early failure in hospitalized patients with CAP. Arch Int Med 2004;164:502-508.

10. Bansal S, Kashyap S, Pal LS, Goel A. a clinical and bacteriological profile of CAP in Shimla, Himachal Pradesh. Ind J Chest Dis Allied Sci 2004;46:17-22.

11. Capoor MR, Nair D, Aggarwal P, Gupta B. rapid diagnosis of CAP using the Bac T/ alert 3D system. Braz J InfectDis 2006; 10:352-6.

12. Shah B A, Singh G, Naik M A, Dhobi G N. Bacteriological and Clinical Profile of CAP in hospitalized patients: Lung India 2010; 27:2.

13. Niederman MS, Bass JB, Campbell GD, Fein AM, Grossman RF, Mandell LA, et al. Guidelines for the initial management of adults with CAP: diagnosis, assessment of severity, and initial antimicrobial therapy. American Thoracic Society. Medical section of the American Lung Association. Am Rev Respir Dis1993;148:1418-1426.

14. Postgraduate Institute of Medical Education and research Chandigarh practice guidelines for the management of community and hospital acquired pneumonias: A consensus statement. Lung India. 2006;23:115-20.

15. Houck PM, Bratzler DW, Nsa W, Ma A, Bartlett JG. Timing of antibiotic administration and outcomes for Medicare patients hospitalized with CAP. Arch Intern Med 2004;164:637-44. 


\section{ORIGINAL ARTICLE}

\begin{tabular}{|l|l|l|l|}
\hline Age & Male & Female & Total \\
\hline $11-20$ & 12 & 8 & 20 \\
\hline $21-30$ & 21 & 16 & 37 \\
\hline $31-40$ & 32 & 22 & 54 \\
\hline $41-50$ & 36 & 42 & 78 \\
\hline $51-60$ & 66 & 34 & 100 \\
\hline $61-70$ & 70 & 48 & 118 \\
\hline $71-80$ & 30 & 13 & 43 \\
\hline & 267 & 183 & 450 \\
\hline Total & & & \\
\hline
\end{tabular}

Table 1:-Distribution of CAP patients

\begin{tabular}{|l|l|l|l|}
\hline & total & Culture positive & Culture negative \\
\hline Prolonged stay & 27 & 11 & 16 \\
\hline DAMA & 11 & 4 & 7 \\
\hline ICU support & 16 & 7 & 9 \\
\hline \begin{tabular}{l} 
Change of antibiotics \\
\hline $\begin{array}{l}\text { Unresolved } \\
\text { pneumonia }\end{array}$
\end{tabular} & 76 & 36 & 40 \\
\hline \begin{tabular}{l} 
Death \\
\hline
\end{tabular} & 24 & 10 & 20 \\
\hline
\end{tabular}

Table 2:- Comparative statistics between culture negative and culture positive CAP patients

\begin{tabular}{|l|l|l|}
\hline Organism isolated & Total & Percentage \\
\hline Streptococcus pneumonia & 54 & 32.7 \\
\hline Klebsiella species & 30 & 18.2 \\
\hline Pseudomonas & 18 & 10.9 \\
\hline Staphylococci & 18 & 10.9 \\
\hline E coli & 9 & 5.4 \\
\hline Acinetobacter & 12 & 7.3 \\
\hline Mixed growth & 9 & 5.4 \\
\hline H influenza & 9 & 5.4 \\
\hline Streptococcus pyogenes & 3 & 1.8 \\
\hline Neisseria meningitides & 3 & 1.8 \\
\hline
\end{tabular}

Table 3:- Microorganism isolated form CAP patients $(n=165)$ 


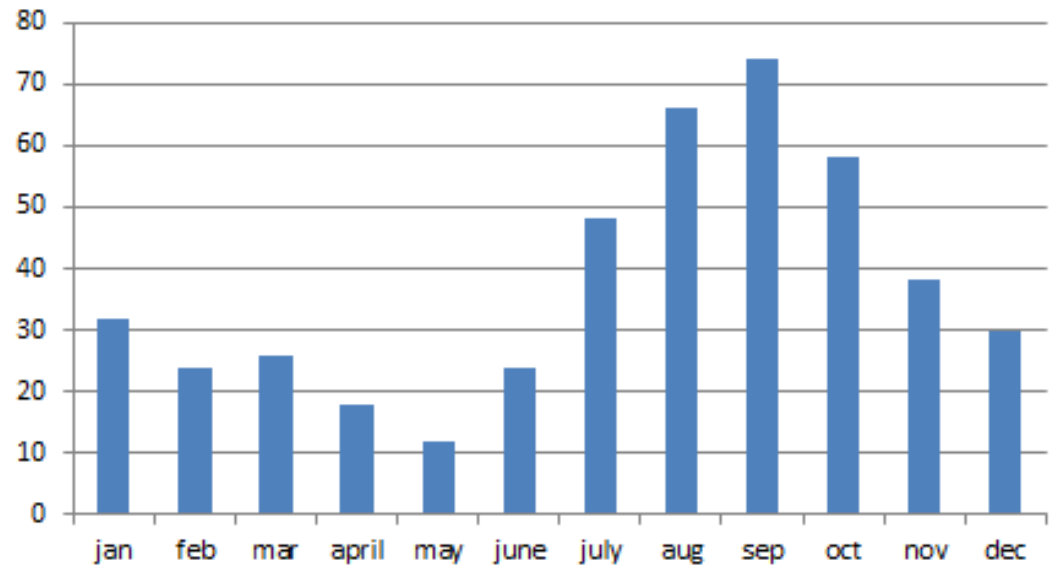

Figure 1:- Graph showing monthly distribution of CAP patients

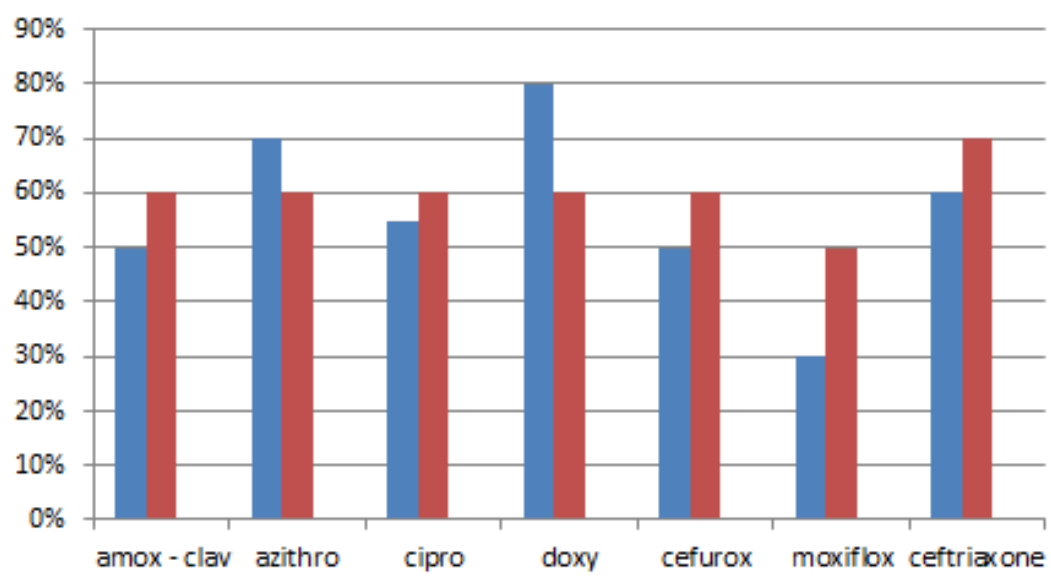

Figure 2:- Resistance profile of S.pneumoniae and S. Aureus

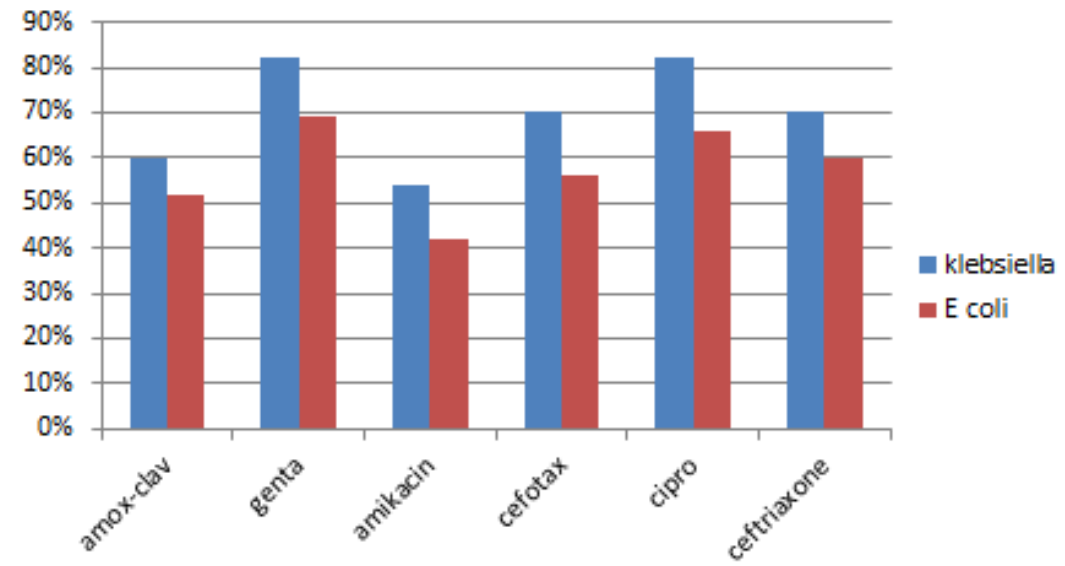

Figure 3:- Resistance profile of Klebsiella and E-coli 


\section{ORIGINAL ARTICLE}

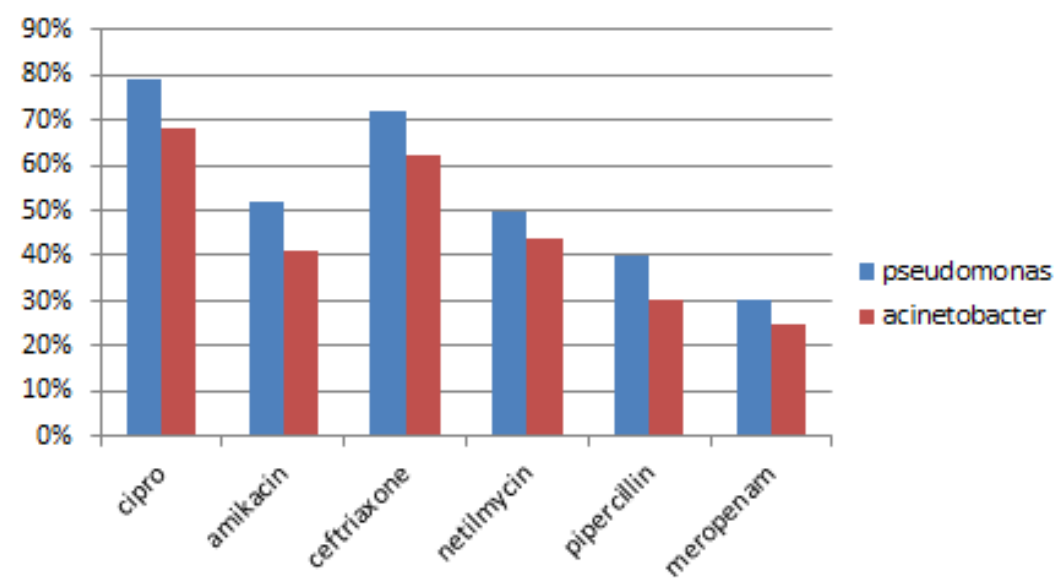

Figure 4:- Resistance profile of Pseudomonas and Acinetobacter 